\title{
Walking without thinking about it
}

\author{
Andrei Herdt \\ Nicolas Perrin \\ Pierre-Brice Wieber
}

\begin{abstract}
We demonstrate how the MPC sheme for the generation of stable bipedal walking motions, that has been introduced in [7], can be further expanded to enhance its flexibility and independency. An algorithm for the control of appropriate orientations of the feet and the trunk permits the robot to turn in a natural and safe way. Polygonal constraints on the positions of the computed feet positions serve to improve its reliability. A logic for the succession of the support phases and an algorithm for the automatic control of their orientations bridge the gap to more autonomy and to more practicability.

Keywords: Walking Humanoid Robot, Linear Model Predictive Control
\end{abstract}

\section{INTRODUCTION}

Walking can be realized only with the help of contact forces between the feet and the ground, and feet can only push on the ground. This involves unilateral contact [1], which limits the motions that a human or robot can realize. In the case of walking on a flat ground, this corresponds to the fact that the Center of Pressure (CoP) can only lie within the support polygon [19]. One class of control laws which can handle such constraints explicitly and efficiently and still generate stable motions is Model Predictive Control (MPC) [10], [11], which maintains a model-based prediction of the future motion of the system on a time horizon which can be of varying length. Such control schemes involve either an objective function which is minimized over the prediction horizon or a constraint on the state of the system at the end of the horizon, or a combination of both. We can observe that the control laws for dynamic walking machines proposed in [2], [6], [7], [9], [8], [12], [13], [15], [14], [16], [17], [18], [20], [21] all belong to this class of MPC schemes, although not presented as such in some cases.

A first category considers only constraints on the state of the system at the end of the horizon, which completely specify the generated motion, such as in [6], [12]. A frequent option is to consider cyclicity at the end of the horizon, in [9], [17], [18]. Another interesting option is to consider that the prediction horizon always ends in a state where the robot is standing still so that we are sure to always have a safe option at hand in case a problem occurs [15]. A downside of these approaches is that being entirely determined by the state of the system at the beginning and end of the horizon, the generated motions may lack some flexibility that could help tackle secondary objectives such as absorbing perturbations efficiently or following precise speed requirements. A relaxation of these constraints has been proposed in [16], but in such a form that the generated motion is still entirely specified by the objective state given at the end of the prediction horizon, resulting in the same lack of flexibility.
A second category considers only an objective function minimized over the prediction horizon, as introduced originally in [8]. A combination of an objective function with constraints at the end of the horizon has been proposed in [13], but this combination is not necessary. It has been shown indeed in [21] that a wide class of objective functions gives rise to stable walking motions without constraints at the end of the horizon, and numerous experiments have shown the real-world efficiency of this approach [14]. This approach introduces a lot of flexibility in the generated motions, which can be used for example to absorb perturbations efficiently [20], dealing explicitly with the constraints on the contact forces. It is therefore this approach that we're going to follow here. As advocated in [10], it is when dealing directly with constraints that MPC schemes really shine. This introduces here inequality constraints in the underlying optimization problem, what can degrade computation time. But what takes time in that case is to discover which inequality constraints hold as equalities at the optimum, and we can hopefully make a good guess about this, and solve this optimization problem with appropriate algorithms in nearly the same time as without inequality constraints [4]

Additional flexibility can also be introduced in the foot step placement [2], what can be used altogether to track precisely any given reference speed [7]. This opens the way to walking without thinking about it: only a desired direction of locomotion needs to be precised and the whole walking motion is taken care of seamlessly, including reactive motions of the feet and CoM in case of perturbations or change of desired direction. This allows introducing for example continuous visual control of the walk [5], but:

- Rotations of the robot haven't been considered so far within these MPC schemes, so we introduce here an MPC scheme generating also rotating motions of the body and feet when required, automatically and safely.

- The safety of the foot step placements generated automatically by the MPC scheme must be ensured. Kinematic feasibility of the steps can be enforced easily as in [15]. We propose here to go a bit further and ensure as well the long-term dynamic feasibility of the steps.

- The time sequence of steps, including start and stop motions, is usually decided externally from the MPC schemes. We propose here a brief formalization of this sequence in the form of a Finite State Machine (FSM), which is incorporated inside the numeric construction of the MPC scheme.

We're going therefore to begin with describing in Sections II and III the MPC scheme originally introduced in [7], 
and present in the following Sections these three additions which allow reaching finally this goal of walking without thinking about it.

\section{KEY ELEMENTS OF A DYNAMIC WALKING MOTION}

\section{A. Motion of the Center of Mass}

Let's consider a frame $\mathcal{C}$ attached to the position of the Center of Mass (CoM) of the robot and to the orientation of its trunk. The position and orientation of this frame will be noted

$$
c=\left(\begin{array}{llllll}
c^{x} & c^{y} & c^{z} & c^{\varphi} & c^{\psi} & c^{\theta}
\end{array}\right),
$$

with Cardan angles $c^{\varphi}, c^{\psi}$ and $c^{\theta}$. The acceleration $\ddot{c}$ of this frame has to be continuous for being realized properly with usual actuators. We'll consider here that it is in fact piecewise linear on time intervals of constant length $\tau$, so with a piecewise constant jerk $\dddot{c}$ on these intervals. The trajectory of this frame over longer time intervals of length $n \tau$ can be obtained simply by integrating in time this piecewise constant jerk together with the initial speed and acceleration. For any coordinate $\alpha \in\{x, y, z, \varphi, \psi, \theta\}$, this leads to simple linear relationships (details can be found in [7])

$$
\begin{aligned}
& C_{i+1}^{\alpha}=S_{p} \hat{c}_{i}^{\alpha}+U_{p} \dddot{C}_{i}^{\alpha}, \\
& \dot{C}_{i+1}^{\alpha}=S_{v} \hat{c}_{i}^{\alpha}+U_{v} \dddot{C}_{i}^{\alpha}, \\
& \ddot{C}_{i+1}^{\alpha}=S_{a} \hat{c}_{i}^{\alpha}+U_{a} \dddot{C}_{i}^{\alpha},
\end{aligned}
$$

where

$$
C_{i+1}^{\alpha}=\left[\begin{array}{c}
c^{\alpha}\left(t_{i+1}\right) \\
\vdots \\
c^{\alpha}\left(t_{i+n}\right)
\end{array}\right], \ldots \dddot{C}_{i+1}^{\alpha}=\left[\begin{array}{c}
\dddot{c}^{\alpha}\left(t_{i+1}\right) \\
\vdots \\
\dddot{c}^{\alpha}\left(t_{i+n}\right)
\end{array}\right]
$$

and

$$
\hat{c}_{i}^{\alpha}=\left[\begin{array}{c}
c^{\alpha}\left(t_{i}\right) \\
\dot{c}^{\alpha}\left(t_{i}\right) \\
\ddot{c}^{\alpha}\left(t_{i}\right)
\end{array}\right] .
$$

\section{B. Motion of the Center of Pressure}

The position $z$ of the Center of Pressure $(\mathrm{CoP})$ on the ground can be approximated by considering only the inertial effects due to the translation of the CoM, neglecting the other effects due to the rotations of the different parts of the robot. This proves to be a very effective approximation, which leads to the simple relationships

$$
\begin{aligned}
& z_{i}^{x}=c_{i}^{x}-\left(c_{i}^{z}-z_{i}^{z}\right) \ddot{c}_{i}^{x} / g, \\
& z_{i}^{y}=c_{i}^{y}-\left(c_{i}^{z}-z_{i}^{z}\right) \ddot{c}_{i}^{y} / g,
\end{aligned}
$$

where the difference $c_{i}^{z}-z_{i}^{z}$ along the $z$ coordinate corresponds to the height of the CoM above the ground, and $g$ is the norm of the gravity force. We'll consider here only the simple case where the height of the CoM above the ground is constant. In that case, we can obtain a relationship similar to (2)-(4):

$$
\begin{aligned}
& Z_{i+1}^{x}=S_{z} \hat{c}_{i}^{x}+U_{z} \dddot{C}_{i}^{x}, \\
& Z_{i+1}^{y}=S_{z} \hat{c}_{i}^{y}+U_{z} \dddot{C}_{i}^{y},
\end{aligned}
$$

with

$$
\begin{aligned}
S_{z} & =S_{p}-\left(c_{i}^{z}-z_{i}^{z}\right) S_{a} / g, \\
U_{z} & =U_{p}-\left(c_{i}^{z}-z_{i}^{z}\right) U_{a} / g .
\end{aligned}
$$

\section{Motion of the feet on the ground}

It has been proposed in [7] to check the feasibility of the motion of the robot only at the instants $t_{i}$. It can be easily shown that this is sufficient even for a time interval $\tau$ as long as $100 \mathrm{~ms}$ in the case of standard walking motions. These $t_{i}$ need however to be synchronized with the contact transitions, when each foot reaches or leaves the ground. Choosing then a time interval $\tau$ corresponding exactly to the duration of the double support, it has been concluded that each time we check the feasibility of the motion at the instants $t_{i}$, only one foot is firmly on the ground. We need therefore to consider only cases with one foot on the ground.

Attaching a frame $\mathcal{F}$ to the foot on the ground, let's consider the current position and orientation $f_{i}$ and the position and orientation of the following steps $\bar{F}_{i+1}$. The positions and orientations $F_{i+1}$ at all instants $t_{i+1} \ldots t_{i+n}$ can be expressed then in a compact form

$$
F_{i+1}^{\alpha}=V_{i+1} f_{i}^{\alpha}+\bar{V}_{i+1} \bar{F}_{i+1}^{\alpha}
$$

for all coordinates $\alpha \in\{x, y, z, \varphi, \psi, \theta\}$ with

$$
V_{i+1}=\left[\begin{array}{c}
1 \\
\vdots \\
1 \\
0 \\
\vdots \\
0 \\
0 \\
\vdots \\
0
\end{array}\right], \quad \bar{V}_{i+1}=\left[\begin{array}{ccc}
0 & 0 & \\
\vdots & \vdots & \\
0 & 0 & \\
1 & 0 & \\
\vdots & \vdots & \\
1 & 0 & \\
0 & 1 & \\
\vdots & \vdots & \\
0 & 1 & \ddots
\end{array}\right],
$$

where the ones simply indicate which sampling time $t_{i+j}$ falls within which step (sampling times corresponding to rows and steps to columns) and therefore which foot position is active at what time.

\section{FOLLOWING A REFERENCE TRANSLATION SPEED}

\section{A. A Model Predictive Control scheme}

It has been proposed in [7] to generate walking motions by directly following a reference speed $\dot{c}_{\text {ref }}$. Only horizontal translations were considered however, and two options were proposed, either regulating the instantaneous speed $\dot{c}(t)$ or the mean speed over two steps. We consider here a combination of these two options. Secondary objectives were also introduced to help obtain a more satisfying behaviour: centering the position of the feet with respect to the position of the CoP, and minimizing the jerk $\dddot{c}(t)$ in order to slightly smooth the resulting trajectory. Taking these objectives into account over a prediction horizon of length $n \tau$, with motions of the CoM, CoP and feet on the ground as introduced in the 
previous Section, we will consider the following optimization problem,

$$
\begin{aligned}
& \min \frac{\alpha}{2}\left\|\dot{C}_{i+1}^{x}-\dot{c}_{r e f}^{x}\right\|^{2}+\frac{\alpha}{2}\left\|\dot{C}_{i+1}^{y}-\dot{c}_{r e f}^{y}\right\|^{2} \\
&+\frac{\beta}{2}\left\|E C_{i+1}^{x}-\dot{c}_{r e f}^{x}\right\|^{2}+\frac{\beta}{2}\left\|E C_{i+1}^{y}-\dot{c}_{r e f}^{y}\right\|^{2} \\
&+\frac{\gamma}{2}\left\|F_{i+1}^{x}-Z_{i+1}^{x}\right\|^{2}+\frac{\gamma}{2}\left\|F_{i+1}^{y}-Z_{i+1}^{y}\right\|^{2} \\
&+\frac{\varepsilon}{2}\left\|\dddot{C}_{i}^{x}\right\|^{2}+\frac{\varepsilon}{2}\left\|\dddot{C}_{i}^{y}\right\|^{2}
\end{aligned}
$$

with a double diagonal matrix

$$
E=\left[\begin{array}{lll}
-I & 0 & I
\end{array}\right] / 2 \tau_{\text {step }}
$$

which computes the mean speed of the CoM over two steps out of $C_{i+1}^{x}$ and $C_{i+1}^{y}$, with $\tau_{\text {step }}$ the duration of a step.

Considering more precisely the motions (2)-(4), (9)-(10) and (13), this optimization problem can be expressed as a canonical QP

$$
\min _{u_{i}} \frac{1}{2} u_{i}^{T} Q_{i} u_{i}+p_{i}^{T} u_{i}
$$

over the vector

$$
u_{i}=\left[\begin{array}{c}
\dddot{C}_{i}^{x} \\
\bar{F}_{i+1}^{x} \\
\dddot{C}_{i}^{y} \\
\bar{F}_{i+1}^{y}
\end{array}\right]
$$

of motion parameters which gathers the jerk $\dddot{C}_{i}$ of the CoM and the future steps $\bar{F}_{i+1}$, with a cyclically varying quadratic term because of the cyclically varying matrix $\bar{V}_{i+1}$ :

$$
Q_{i}=\left[\begin{array}{cc}
Q_{i}^{\prime} & 0 \\
0 & Q_{i}^{\prime}
\end{array}\right]
$$

$$
\begin{aligned}
& Q_{i}^{\prime}= \\
& {\left[\begin{array}{cc}
\alpha U_{v}^{T} U_{v}+\beta U_{p}^{T} E^{T} E U_{p}+\gamma U_{z}^{T} U_{z}+\varepsilon I & -\gamma U_{z}^{T} \bar{V}_{i+1} \\
-\gamma \bar{V}_{i+1}^{T} U_{z} & \gamma \bar{V}_{i+1}^{T} \bar{V}_{i+1}
\end{array}\right]}
\end{aligned}
$$

and

$$
\begin{gathered}
p_{i}= \\
{\left[\begin{array}{c}
\alpha U_{v}^{T}\left(S_{v} \hat{c}_{i}^{x}-\dot{c}_{r e f}^{x}\right)+\beta U_{p}^{T} E^{T}\left(E S_{p} \hat{c}_{i}^{x}-\dot{c}_{r e f}^{x}\right)+ \\
\gamma U_{z}^{T}\left(S_{z} \hat{c}_{i}^{x}-V_{i+1} f_{i}^{x}\right) \\
-\gamma \bar{V}_{i+1}^{T}\left(S_{z} \hat{c}_{i}^{x}-V_{i+1} f_{i}^{x}\right) \\
\alpha U_{v}^{T}\left(S_{v} \hat{c}_{i}^{y}-\dot{c}_{r e f}^{y}\right)+\beta U_{p}^{T} E^{T}\left(E S_{p} \hat{c}_{i}^{y}-\dot{c}_{r e f}^{y}\right)+ \\
\gamma U_{z}^{T}\left(S_{z} \hat{c}_{i}^{y}-V_{i+1} f_{i}^{y}\right) \\
-\gamma \bar{V}_{i+1}^{T}\left(S_{z} \hat{c}_{i}^{y}-V_{i+1} f_{i}^{y}\right)
\end{array}\right]}
\end{gathered}
$$

\section{B. Constraints on the Center of Pressure}

Since the feet of the robot can only push on the ground, the $\mathrm{CoP}$ can lie only within the support polygon, the convex hull of the contact points between the feet and the ground [19]. Any trajectory not satisfying this constraint can't be realized properly, so this needs to be taken into account when computing a walking motion with the MPC scheme (15). Following [7], we'll consider that the foot on the ground has a polygonal shape, potentially depending on the support foot $s \in\{$ Left, Right $\}$, so that this constraint can be expressed as a set of linear constraints on the position of the CoP:

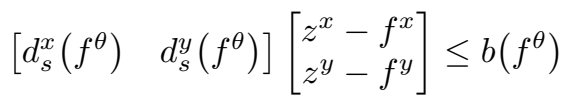

which are linear with respect to the position $\left(f^{x}, f^{y}\right)$ of the foot on the ground but nonlinear with respect to its orientation $f^{\theta}$. The column vectors $d^{x}$ and $d^{y}$ gather the $x$ and $y$ coordinates of the normal vectors to the edges of the feet and the column vector $b$ corresponds to the positioning of these edges.

Considering this constraint at all instants $t_{i+1} \ldots t_{i+n}$ can be expressed with the help of (13) as

$$
D_{i+1}\left[\begin{array}{l}
Z_{i+1}^{x}-V_{i+1} f_{i}^{x}-\bar{V}_{i+1} \bar{F}_{i+1}^{x} \\
Z_{i+1}^{y}-V_{i+1} f_{i}^{y}-\bar{V}_{i+1} \bar{F}_{i+1}^{y}
\end{array}\right] \leq b_{i+1},
$$

with the simple double block-diagonal matrix

$$
\begin{aligned}
& D_{i+1}= \\
& {\left[\begin{array}{ccccc}
d_{s_{i+1}}^{x}\left(f_{i+1}^{\theta}\right) & & 0 & d_{s_{i+1}}^{y}\left(f_{i+1}^{\theta}\right) & 0 \\
& \ddots & & & \ddots \\
0 & & d_{s_{i+n}}^{x}\left(f_{i+n}^{\theta}\right) & 0 & d_{s_{i+n}}^{y}\left(f_{i+n}^{\theta}\right)
\end{array}\right]}
\end{aligned}
$$

and the vector

$$
b_{i+1}=\left[\begin{array}{c}
b\left(f_{i+1}^{\theta}\right) \\
\vdots \\
b\left(f_{i+n}^{\theta}\right)
\end{array}\right] .
$$

With respect to the vector $u_{i}$ introduced in (18), this constraint takes the following form

$$
\begin{aligned}
D_{i+1}\left[\begin{array}{cccc}
U_{z} & -\bar{V}_{i+1} & 0 & 0 \\
0 & 0 & U_{z} & -\bar{V}_{i+1}
\end{array}\right] u_{i} \\
\quad \leq b_{i+1}+D_{i+1}\left[\begin{array}{l}
V_{i+1} f_{i}^{x}-S_{z} \hat{c}_{i}^{x} \\
V_{i+1} f_{i}^{y}-S_{z} \hat{c}_{i}^{y}
\end{array}\right],
\end{aligned}
$$

which can be introduced directly in the QP (17).

\section{FOLLOWING A REFERENCE ROTATION SPEED}

Considering also the rotations of the frame $c$ around the vertical axis $z$ in (15) can be done straightforward by employing the same linear relationships (2)-(4) to formulate the following additional objective:

$$
\min \frac{\alpha}{2}\left\|\dot{C}_{i+1}^{\theta}-\dot{c}_{r e f}^{\theta}\right\|^{2}+\frac{\beta}{2}\left\|E C_{i+1}^{\theta}-\dot{c}_{r e f}^{\theta}\right\|^{2}
$$

When rotating the robot has to take care of appropriate orientations of the feet that have to follow the rotating trunk in one way or another. Since the most efficient walk is in the sagittal direction the feet should be aligned with the trunk the most of the time. We propose therefore the following optimization problem that minimizes the squared sum of the 
angular deviations between the feet and the trunk for the whole preview period:

$$
\begin{gathered}
\min \frac{\gamma}{2}\left\|\sum_{i}\left(f_{i}^{\theta}-c_{i}^{\theta}\right)\right\|^{2} \\
{\left[\begin{array}{cc}
E_{L} & 0 \\
0 & E_{R}
\end{array}\right]\left[\begin{array}{l}
\dot{F}_{i+1}^{\prime \theta, L} \\
\dot{F}_{i+1}^{\prime \theta,}, R
\end{array}\right]=0}
\end{gathered}
$$

with $\dot{F}_{i+1}^{\prime \theta}=S_{v} \hat{f}_{i}^{\theta}+U_{v} \dddot{F}_{i}^{\theta}$ the complete trajectory of the left or the right foot and $E_{R / L}$ the selection matrices that select all elements of $\dot{F}_{i+1}^{\prime \theta}$ that fall into the support time of the corresponding foot.

Naturally, aligning the feet as much as possible with the trunk reduces also the probability of a violation of physical constraints but it might not be enough so that we can add hard constraints to the above QP:

$$
\begin{gathered}
\left\|F_{i+1}^{\prime \theta, R}-F_{i+1}^{\prime \theta, L}\right\|<\theta_{\max }^{r l} \\
\left\|F_{i+1}^{\prime \theta}-C_{i+1}^{\theta}\right\|<\theta_{\max }^{F T} \\
\left\|\dot{F}_{i+1}^{\prime \theta}-\dot{C}_{i+1}^{\theta}\right\|<\dot{\theta}_{\max }^{F T} \\
\left\|\ddot{F}_{i+1}^{\prime \theta}-\ddot{C}_{i+1}^{\theta}\right\|<\ddot{\theta}_{\max }^{F T} .
\end{gathered}
$$

The constraint (30) would limit the intersection angles of the feet to prevent self collisions. Constraints (31)-(33) would limit the angles, the velocity and the acceleration of the feet with respect to the trunk.

Contrary to the translational motions of the feet (section IIC) we use for their orientations the relationships (2)-(4) instead of (13). Thus we can generate smoother trajectories of the feet orientations and their derivatives and to apply the constraints (31)-(33) also for the feet in the air.

As mentioned in section III-B, including the orientations $f^{\theta, s}$ of the feet in contact with the ground as free variables would result in nonlinear CoP constraints (22). In order to keep the linear form of the original QP we choose not to extend it but to predetermine the orientations of the feet and the trunk by solving the above QP prior to (15).

\section{CONSTRAINTS ON THE FOOT PLACEMENT}

We need to assure that the footsteps decided by (15) are feasible with respect to joint angles and speed limitations, self-collision and over-stretching avoidance and similar geometric and kinematic limitations. What we need to do, in order to keep the Linear MPC structure of our algorithm, is to derive simple approximations of all these limitations that can be expressed in the form of linear constraints on the vector $u_{i}$ defined in III-A.

We can derive for example simple linear bounds on the positions of the feet one with respect to the other with minimum and maximum values preventing collision on one side and over-stretching of the legs on the other side. Precisely, we are willing to define simple polygonal limitations for the placement of one foot relative to the other, what can similiarly to (23) be expressed as a set of linear constraints

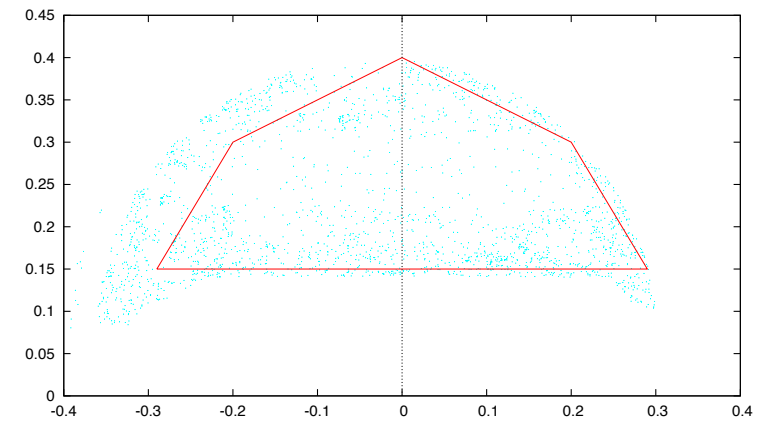

Fig. 1. Polygonal approximation of the feasible reagion of the final position of one foot relative to the support foot.

of this form:

$$
A_{j+1}=\left[\begin{array}{rrrrrr}
-11 & & 0 & -11 & & 0 \\
& \ddots & & & \ddots & \\
0 & & -11 & 0 & & -11
\end{array}\right]\left[\begin{array}{c}
f_{i}^{x} \\
\bar{F}_{i+1}^{x} \\
f_{i}^{y} \\
\bar{F}_{i+1}^{y}
\end{array}\right] \leq b_{j+1}
$$

with

$$
\begin{aligned}
& A_{j+1}=
\end{aligned}
$$

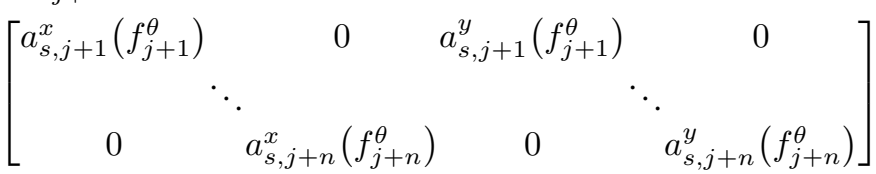

To do so, we took in consideration the MPC scheme in [8] already implemented on the HRP-2 robot. Although the regions of feasibility obviously differ, at least slightly, from one MPC to another, what we are looking for is simply a relevant initial heuristic. Later on, if we apply our scheme to HRP-2, we can observe the effects of the initial heuristic, and thus start a hopefully converging sequence of modifications of the polygon, in order to get one more suited to our own Linear MPC structure.

Starting from the standard initial position of HRP-2, we used extensive offline calculations to build a point cloud of positions that can be reached by the center of the left foot with one single step.

The offline computation involved random positions which were used as inputs by the MPC scheme in [8] to generate joint space trajectories. Each trajectory was then checked with an ad hoc verification process which declared feasible a trajectory when it does not violate joint limits, avoids selfcollisions and respects some other dynamic constraint.

The point cloud on Fig. 1 shows all the positions that lead to feasible trajectories. It reveals what seems to be a safe area of quite simple geometry. We define a 5 edges polygon included in this area, as shown on Fig. 1. It is 
symmetric about the y-axis in order not to take into account specificities of the HRP-2 robot or the MPC scheme inv [8] that tend to result in a slightly larger feasible zone when walking backwards.

Concerning the maximum joint speed, we have found that a very simple bound on the position of the next foot step depending on the current position of the foot in the air and a simple Cartesian maximum speed gives good results:

$$
B\left(\begin{array}{l}
\left(F_{i}^{x}\right)_{1}-f^{x}(t) \\
\left(F_{i}^{y}\right)_{1}-f^{y}(t)
\end{array}\right) \leq\left(t_{\text {touchdown }}-t\right) v_{\max }
$$

with $\left(F_{i}^{x}, F_{i}^{y}\right)_{1}$ the position of the next foot step, $\left(f^{x}(t), f^{y}(t)\right)$ the current position of the foot in the air, $v_{\max }$ a vector of approximate maximum Cartesian speed in the directions indicated by the matrix $B$ and $t_{\text {touchdown }}$ the time when the foot in the air is planned to touch the ground.

\section{THE LOGIC OF WALKING}

In order to avoid nonlinearities, the MPC scheme introduced in [7] takes into account only simple sipports. Because of a sampling synchronized with the contact transitions (see II-C) this is valable during walking but restricting during a stop. To correct this deficiency we propose to stop only with the feet aligned in the laterale plane of the robot. The double support phase can then be smoothly integrated in the computation of the CoP constraints due to its rectangular form, $s \in\{L, R, D\}$. The assembling of the constraints and the matrices $V_{i+1}, \bar{V}_{i+1}, E_{R}, E_{L}$ requires for each instant of the whole preview period prior knowledge about the corresponding support phase $s_{i}$. This knowledge can be provided by the finite state machine (FSM) in Fig. 2. Every state of this FSM corresponds thus to a vector $\left(d_{x}^{s}\left(f^{\theta}\right), d_{y}^{s}\left(f^{\theta}\right)\right)$ and a nonzero entry either in $\bar{V}_{i+1}$ or $V_{i+1}$ and $E_{R}$ or $E_{L}$ depending on the step number and the previewed sampling time. Given a constant speed reference for the whole preview window the switching process is defined only by the predefined lengths of the simple and double support phases.

A fixed double support phase can require to effectuate one step before the stop in a double support phase, which led to the introduction of the supplementary parameter $s l$ which defines the number of steps to be done before a stop (Fig. 2 , right).

\section{Simulation RESUlts}

Fig. 3 shows a walking gait generated by our MPC scheme when the system is not submitted to any perturbation. The robot starts from rest in a double support phase which is limited to $0.4 \mathrm{~s}$ and walks continuously for $20 \mathrm{~s}$, making a step regularly every $0.8 \mathrm{~s}$. $0.4 \mathrm{~s}$ after the switch of the reference velocity to $0.25 \frac{\mathrm{m}}{\mathrm{s}}$ the robot starts to walk forward by lifting its right foot first. Five seconds later the reference speed vector is instantly turned by 90 degrees to the right. At the beginning of the tenth second the reference is switched again to $0.25 \frac{\mathrm{m}}{\mathrm{s}}$ forward and an additional rotational reference speed of $-\frac{\pi}{20} \frac{\mathrm{deg}}{\mathrm{s}}$ is applied. Finally all references are removed and the robot passes after one additional step
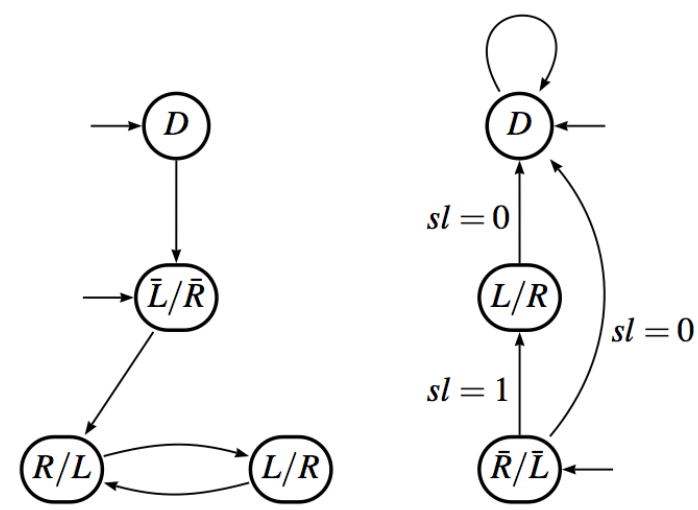

Fig. 2. Evolution of CoP constraints for $\|\dot{X}\|>0$ (left) and $\|\dot{X}\|=0$ (right) as a finite state machine.

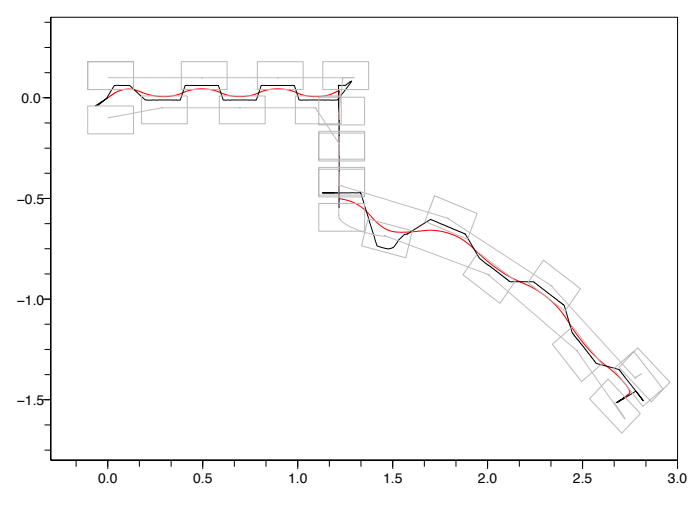

Fig. 3. Motion of the CoM (in red) and CoP (in black) and foot steps generated by our MPC scheme when the system is not submitted to any perturbation. Note an evolution of the CoP from heels to toes very similar to what can be observed in humans.

into double support with the feet aligned in the same lateral plane and with equal angles. We can see that the QP together with the additional control layers, introduced in the last three sections, manage all requirements including the turning motion and the stop in a double support phase. Especially, when a rotation is desired the algorithm proves to be able to find appropriate feet angles during walking and for the predetermined double support constraints.

Fig. 4 shows the forward speed of the CoM for three different demanded saggital velocities. We can observe that the QP manages to perfectly realize the desired motion for velocities slower than $0.3 \frac{\mathrm{m}}{\mathrm{s}}$. For slightly bigger references oscillations occur but it still manages to maintain the desired mean value. These oscillations occur when step length become such that successive support polygons do not superpose in the saggital plane. Consequently, the Center of Pressure cannot permanently share its lateral plane with the Center of Mass but has to deviate which results in forward acceleration of the CoM. During the next support phase this acceleration has not only to be absorbed but the CoM has to be decelerated so that the desired mean value can be maintained in the 
Sagittal velocity $[\mathrm{m} / \mathrm{s}]$

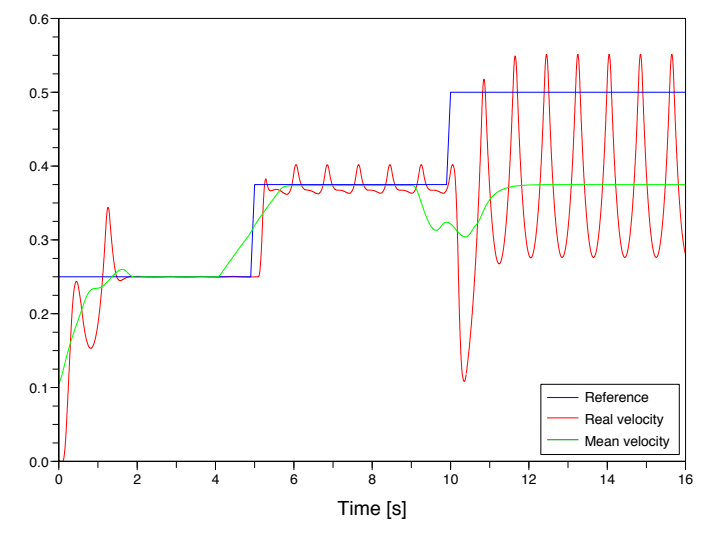

Fig. 4. Forward speed of the CoM (red) for three different reference velocities (blue).

best possible way. Constraints on the feet positions together with fixed support lengths limit finally the highest possible speed that the QP is able to realize when a too big velocity reference is applied, as can be seen on the right side of Fig. 4 . However, this last part demonstrates one of the most valuable properties of this walking motion generation scheme: safety prevails, in the sense that the generated motion is always kept feasible, even if that means not realizing the desired motion. Here, the goal of the robot is to move forward at a certain speed, but this goal is fulfilled only as much as possible.

To demonstrate the behavior of the feet orientations control layer introduced in section IV we simulated situations where at least one of the angular constraints would be violated for a trunk that changes its velocity instantly. We can see on the left half of Fig. 5 that due to the angular velocity constraint on the feet the robot has to make several steps before being able to effectively minimize the angular deviations between the feet and the trunk. The right support foot on the contrary can not achieve this goal as its angle towards the left foot is limited. None of these violations does affect the rotation of the trunk, contrary to the following decreasing slope where the robot instantly changes its rotation to follow a higher negative reference. Because it has been previewed that the constraint on the angle between the trunk and at least one previewed foot would be violated, the rotation speed of the trunk has been reduced by (31). This behavious represents in our view a good trade-off between reliability and performance as the rotation rate of the robots trunk is reduced only when necessary to prevent violations of constraints.

Fig. 6 depicts the feet configurations that are obtained when the robot is supposed to turn around itself. We can see that the algorithm aims to bring an edge of the feet as close as possible to the Center of Mass. Although it looks unnatural this behavior is a logical consequence of the overall objective, which is in this case also the immobilisation of the Center of Mass. By bringing the edges as close as possible to the position of the CoM the QP minimizes the deviation

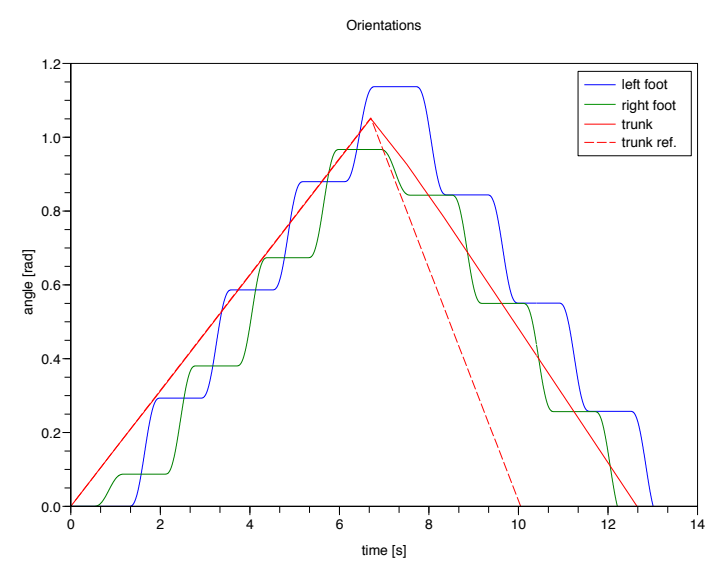

Fig. 5. Orientations of the trunk (red, solid) and the feet (blue and green) for a rotation reference of the trunk which would result in the dashed curve, supposed that the trunk can instantly change its velocity.

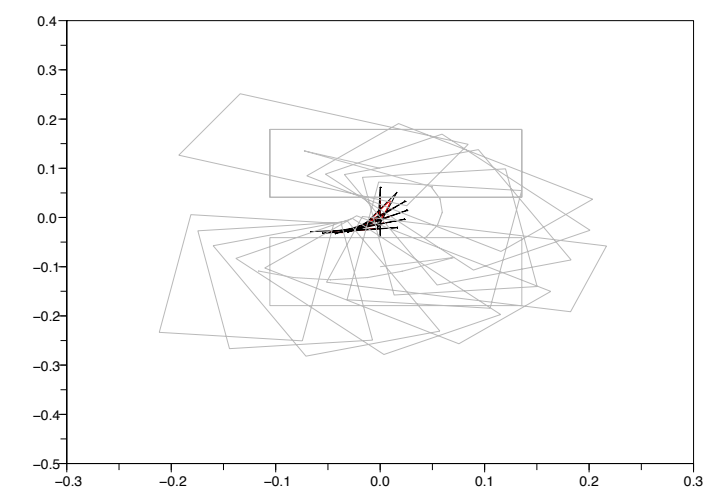

Fig. 6. Motion of the CoM (in red) and CoP (in black) and foot steps generated by our MPC scheme when only a rotational speed is given.

from zero velocity as much as possible.

With $\mathrm{N}=16$ time intervals of length $\mathrm{T}=0.1 \mathrm{~s}$, the prediction horizon is $\mathrm{NT}=1.6 \mathrm{~s}$, what corresponds to two steps. Assembling and solving the full QP (18) in this case takes less than $1 \mathrm{~ms}$ in average with state of the art solvers, notwithstanding the possible optimizations presented in [3] or the optimized solver presented in [4] which can help to reduce furthermore the computation time.

\section{CONCLUSION}

Lacking important features on the side of the reliability of the resulting motions as well as the flexibility of control this last modification needed to be completed in order to unfold its full potential. We have therefore defined approximations of admissible zones for the positionning of the feet and we added an inner logic for their succession. An additional layer on top of the original QP contributes a third degree of freedom to the control. The resulting algorithm has been successfully implemented and tested for visual servoing tasks in [5]. 
Lateral velocity $[\mathrm{m} / \mathrm{s}]$

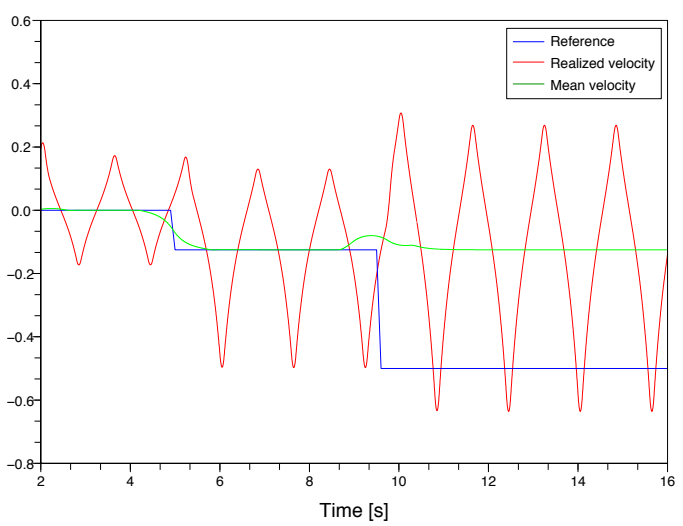

Fig. 7. Lateral speed of the CoM (red), mean value of this speed over prediction horizons (grey) and reference speed (blue). We can see, that because of the unavoidable lateral sway motion, only the desired mean value of the lateral CoM speed can be maintained.

\section{REFERENCES}

[1] B. Brogliato. Nonsmooth Impact Mechanics. Springer-Verlag, 1999.

[2] H. Diedam, D. Dimitrov, P.-B. Wieber, K. Mombaur, and M. Diehl. Online walking gait generation with adaptive foot positioning through linear model predictive control. In Proceedings of the IEEE/RSJ International Conference on Intelligent Robots \& Systems, pages 1121-1126, Nice, 2008.

[3] D. Dimitrov, J. Ferreau, P.-B. Wieber, and M. Diehl. On the implementation of model predictive control for on-line walking pattern generation. In Proceedings of the IEEE International Conference on Robotics \& Automation, 2008.

[4] D. Dimitrov, O. Wieber, P.-B. Stasse, J. Ferreau, and H. Diedam. An optimized linear model predictive control solver for online walking motion generation. In Proceedings of the IEEE International Conference on Robotics \& Automation, 2009.

[5] C. Dune, A. Herdt, O. Stasse, and P-B. Wieber. Canceling the sway motion in visual servoing of dynamic walking. In Submitted to IEEE/RSJ International Conference on Intelligent Robots \& Systems, 2010.

[6] K. Harada, S. Kajita, K. Kaneko, and H. Hirukawa. An analytical method on real-time gait planning for a humanoid robot. International Journal of Humanoid Robotics, 18(8):816-830, 2008.

[7] A. Herdt, H. Diedam, P.B. Wieber, D. Dimitrov, K. Mombaur, and M. Diehl. Online walking motion generation with automatic foot step placement. Advanced Robotics, 24(6), 2010.

[8] S. Kajita, F. Kanehiro, K. Kaneko, K. Fujiwara, K. Harada, K. Yokoi, and $\mathrm{H}$. Hirukawa. Biped walking pattern generation by using preview control of zero-moment point. In Proceedings of the IEEE International Conference on Robotics \& Automation, 2003.

[9] S. Kajita, O. Matsumoto, and M. Saigo. Real-time 3d walking pattern generation for a biped robot with telescopic legs. In Proceedings of the 2001 IEEE International Conference on Robotics \& Automation, 2001.

[10] D.Q. Mayne. Control of constrained dynamic systems. European Journal of Control, 7:87-99, 2001.

[11] M. Morari and J.H. Lee. Model predictive control: past, present and future. Computers and Chemical Engineering, 23:667-682, 1999.

[12] M. Morisawa, K. Harada, S. Kajita, K. Kaneko, F. Kanehiro, K. Fujiwara, S. Nakaoka, and H. Hirukawa. A biped pattern generation allowing immediate modification of foot placement in real-time. In Proceedings of the International Conference on Humanoid Robotics, 2006.

[13] K. Nagasaka, Y. Kuroki, S. Suzuki, Y. Itoh, and J. Ymagaguchi. Integrated motion control for walking, jumping and running on a small bipedal entertainment robot. In Proceedings of the IEEE International Conference on Robotics \& Automation, 2004.

[14] K. Nishiwaki and S. Kagami. Online walking control systems for humanoids with short cycle pattern generation. International Journal of Robotics Research, 28(6):729-742, 2009.

[15] K. Nishiwaki, S. Kagami, Y. Kuniyoshi, M. Inaba, and H. Inoue. Online generation of humanoid walking motion based on a fast generation method of motion pattern that follows desired zmp. In Proceedings of the IEEE/RSJ International Conference on Intelligent Robots \& Systems, 2002.

[16] T. Sugihara and Y. Nakamura. A fast online gait planning with boundary condition relaxation for humanoid robots. In Proceedings of the 2005 IEEE International Conference on Robotics and Automation, 2005.

[17] R. Tajima, Honda D., and K. Suga. Fast running experiments involving a humanoid robot. In Proceedings of the IEEE International Conference on Robotics \& Automation, 2009.

[18] T. Takenaka, T. Matsumoto, and T. Yoshiike. Real time motion generation and control for biped robot -1st report: Walking gait pattern generation-. In Proceedings of the IEEE/RSJ International Conference on Intelligent Robots \& Systems, 2009.

[19] P.-B. Wieber. On the stability of walking systems. In Proceedings of the International Workshop on Humanoid and Human Friendly Robotics, 2002.

[20] P.-B. Wieber. Trajectory free linear model predictive control for stable walking in the presence of strong perturbations. In Proceedings of the International Conference on Humanoid Robotics, 2006.

[21] P.-B. Wieber. Viability and predictive control for safe locomotion. In Proceedings of the IEEE/RSJ International Conference on Intelligent Robots \& Systems, 2008. 